\title{
Inhibiting PLK1 induces autophagy of acute myeloid leukemia cells via mammalian target of rapamycin pathway dephosphorylation
}

\author{
YAN-FANG TAO $^{1 *}$, ZHI-HENG LI $^{1 *}$, WEI-WEI DU ${ }^{2 *}$, LI-XIAO XU $^{1}$, JUN-LI REN $^{2}$, XIAO-LU LI $^{1}$, \\ FANG FANG $^{1}$, YI XIE ${ }^{1}$, MEI LI ${ }^{1}$, GUANG-HUI QIAN ${ }^{1}$, YAN-HONG LI ${ }^{1}$, YI-PING LI ${ }^{1}$, GANG LI ${ }^{1}$, \\ YI WU ${ }^{1}$, XING FENG ${ }^{1}$, JIAN WANG ${ }^{1}$, WEI-QI HE ${ }^{3}$, SHAO-YAN HU ${ }^{2}$, JUN LU ${ }^{2}$ and JIAN PAN ${ }^{1}$ \\ ${ }^{1}$ Institute of Pediatrics and ${ }^{2}$ Department of Hematology and Oncology, Children's Hospital of Soochow University, \\ Suzhou, Jiangsu 215003; ${ }^{3}$ CAM-SU Genomic Resource Center, Soochow University, Suzhou, Jiangsu 215123, P.R. China
}

Received September 1, 2016; Accepted January 30, 2017

DOI: $10.3892 /$ or.2017.5417

\begin{abstract}
Decreased autophagy is accompanied by the development of a myeloproliferative state or acute myeloid leukemia (AML). AML cells are often sensitive to autophagy-inducing stimuli, prompting the idea that targeting autophagy can be useful in AML cytotoxic therapy. AML NB4 cells overexpressing microtubule-associated protein 1 light chain 3-green fluorescent protein were screened with 69 inhibitors to analyze autophagy activity. AML cells were treated with the polo-like kinase 1 (PLK1) inhibitors RO3280 and BI2536 before autophagy analysis. Cleaved LC3 (LC3-II) and the phosphorylation of mammalian target of rapamycin (mTOR), adenosine monophosphate-activated protein kinase, and Unc-51-like kinase 1 during autophagy was detected with western blotting. Autophagosomes were detected using transmission electron microscopy. Several inhibitors had promising autophagy inducer effects: BI2536, MLN0905, SK1-I, SBE13 HCL and RO3280. Moreover, these inhibitors all targeted PLK1. Autophagy activity was increased in the NB4 cells treated with RO3280 and BI2536. Inhibition of PLK1 expression in NB4, K562 and HL-60 leukemia cells with RNA interference increased LC3-II and autophagy activity. The phosphorylation of mTOR was reduced significantly in NB4 cells treated with RO3280 and BI2536, and was also
\end{abstract}

Correspondence to: Professor Jun Lu, Department of Hematology and Oncology, Children's Hospital of Soochow University, Suzhou, Jiangsu 215003, P.R. China

E-mail: drlujun_sz@163.com

Professor Jian Pan, Institute of Pediatrics, Children's Hospital of Soochow University, Suzhou, Jiangsu 215003, P.R. China

E-mail: panjian2008@163.com

${ }^{*}$ Contributed equally

Key words: BI2536, RO3280, autophagy, mTOR phosphorylation, pediatric acute myeloid leukemia, polo-like kinase 1 reduced significantly when PLK1 expression was downregulated in the NB4, K562 and HL-60 cells. We demonstrate that PLK1 inhibition induces AML cell autophagy and that it results in mTOR dephosphorylation. These results may provide new insights into the molecular mechanism of PLK1 in regulating autophagy.

\section{Introduction}

Autophagy, or autophagocytosis, is from the Greek auto-, 'self', and phagein, 'to eat'. Autophagy is a conserved protein degradation pathway that plays important roles in mammalian cell survival, proliferation and differentiation, particularly within the hematopoietic system. Diminished autophagic flux results in the development of a myeloproliferative state or acute myeloid leukemia (AML) (1). In AML, there is copy number loss in autophagic genes such as BECN1 $(2,3), A T G 7(1,4)$ and ATG5 (1,5). Decreased autophagy and the development of AML are related. BECN1 is a critical mediator that influences the onset and progress of autophagy, and there is a remarkable association between reduced $B E C N 1$ expression and FLT3-internal tandem duplication (ITD) mutation (6). ATG7 and ATG5 play important roles in autophagy and their loss of function in hematopoietic stem and progenitor cells (HSPCs) always leads to a lethal pre-leukemic phenotype in mice (1).

Recently, autophagy pathway inducers have shown promising effects for treating AML. Mammalian target of rapamycin (mTOR) signaling is a critical pathway in the biology of several cancers, including AML. Constitutive activation of the phosphatidylinositol 3-kinase (PI3K)/mTOR signaling pathway has been observed in different cancers and leukemias, including chronic myelogenous leukemia (CML), AML and acute lymphoblastic leukemia (ALL). The PI3K/mTOR pathway has always been considered a prosurvival factor in leukemia stem cells and leukemic precursors, and its inhibition has been regarded as an effective therapeutic approach (7). MLN0128 is a novel, recently developed mTOR kinase inhibitor that can disrupt survival signaling and triggers apoptosis in AML stem and AML progenitor cells (8). Abnormal mTOR activity contributes to chemotherapy resistance, and aberrant activation 
of the PI3K/mTOR pathway promotes sorafenib resistance in AML cells (9).

The serine/threonine protein kinase polo-like kinase 1 (PLK1), or serine/threonine-protein kinase 13 (STPK13), regulates multiple intracellular processes, including DNA replication, mitosis and stress response. PLK1 is expressed during mitosis and is overexpressed in multiple cancers, including breast cancer (10), prostate cancer (11), renal cancer (12) and neuroblastoma (13). PLK1 is also highly expressed in leukemia cell lines; PLK1 expression in patients with AML is significantly higher than in normal progenitors (14). Furthermore, PLK1 expression in normal or untransformed cells is much lower than in cancer cells, which renders PLK1 a suitable anticancer target $(15,16)$. Downregulating or inhibiting the kinase activity of PLK1 induces cell cycle arrest and apoptosis in most cancer cell types in vitro and in vivo (17-20). The potential of PLK1 inhibitors as cancer therapeutics has been investigated widely. The PLK1 inhibitor volasertib has shown considerable promise in clinical studies of AML, having reached phase III trials $(21,22)$. Other PLK1 inhibitors, including GSK461364A, TKM-080301, GW843682, purpurogallin and poloxin are in early clinical development (23).

To date, the molecular function of PLK1 in AML cell autophagy is unclear. In our study, the autophagy-related effect of PLK1 was evaluated in AML cells to characterize its preclinical efficacy.

\section{Materials and methods}

Animal and human rights statement. The studies have been performed in accordance with the ethical standards as laid down in the 1964 Declaration of Helsinki and its later amendments. Ethical approval was provided by the Children's Hospital of Soochow University Ethics Committee (nos. SUEC2008-011 and SUEC2000-021).

Cell and culture conditions. Leukemia cell lines HL-60 and K562 were obtained from the American Type Culture Collection (ATCC, Manassas, VA, USA). NB4 cell line (gifts from Hematology Institute of Soochow University). All cell lines were maintained at $37^{\circ} \mathrm{C}$ in the RPMI-1640 (Gibco Life Technologies, Carlsbad, CA, USA) supplemented with $10 \%$ fetal bovine serum (FBS; Invitrogen Life Technologies, Carlsbad, CA, USA). Sixty-nine inhibitors ABT-263, ABT-737, YM155, SK1-I, SKI-5C, 17-AAG, XAV-939, AC220, tosedostat (CHR2797), VER-50589, FH535, G-749, BV-6 (apoptosis and anti-apoptosis); rapamycin, valproic acid, 3-methyladenine (3-MA), BEZ235, HS-173, pilaralisib (autophagy); SP600125, elesclomol, BAY 11-7082, ipatasertib, SB202190, PD98059, LY294002, INCB018424, SH-4-54, AT13148, JNK inhibitor IX, PX-478 $2 \mathrm{HCl}$ (oxidative stress and MAPK pathway); BI 2536, PF-3758309, nutlin-3, MI-773, YH239-EE, XL-413, MLN0905, SBE13 HCL, RO3280, volasertib, nutlin-3b (cell cycle); JIB-04, GSK J1, GSK J4, GSK 126, LBH589, SGC-CBP30, 4SC-202 (histone modification); KPT-276, KPT-330, KPT-185, KPT-335 (CRM1); CW069, TAPI-1, INH6, ODM-201, ESI-09, EW-7197, GDC-0623, AZD6738, LY3009120, SB-3CT, INH1, XMD8-92, LY2584702, ML323 (other targets). Tosedostat (CHR2797) was purchased from Molbase Chemicals (Shanghai, China). Other inhibitors were purchased all from Selleck Chemicals (West Paterson, NJ, USA) and was dissolved in dimethyl sulfoxide (DMSO) (cat. no. D4540; Sigma-Aldrich, St. Louis, MO, USA).

Expression of hLC3 in leukemia cells with UBC-LC3-GFP lentivirus. UBC-LC3-GFP lentivirus was purchased from Shanghai Genechem Co., Ltd. (Shanghai, China) (24). Lentivirus infection was according to the manufacturer (Shanghai GeneChem Co, Ltd.) at a final concentration of 100-200 multiplicity of infection (MOI). Aggregation of LC3 was observed when the autophagy occurred in cells. LC3-GFP fusion protein is diffused in the cytoplasm when the cells occur autophagy. LC3-GFP fusion protein translocates to autophagic membranes, forming a bright green fluorescence spots under the fluorescence confocal microscopy (Olympus; Olympus Corporation, Tokyo, Japan) when the cells are autophagic, a spot is equivalent to an autophagosome thus can be counted to evaluate the level of autophagy activity.

Transmission electron microscopy (TEM). The presence of autophagosomes in TEM is the standard for detecting autophagy. TEM analysis protocol was according to a previous report (25). Briefly, leukemia cells grown on 6-well plates were treated with $\mathrm{RO} 3280, \mathrm{BI} 2536$ or the same volume of DMSO and $4 \mathrm{~h}$ later were harvested and washed with phosphate-buffered saline (PBS) prior to fixing in fixative buffer. Subsequently, cells were collected and suspended with $2.5 \%$ glutaraldehyde. Then treated with $2 \%$ osmium tetroxide in $0.1 \mathrm{M}$ sodium cacodylate buffer, and embedded in resin. Finally, the samples were sliced and processed for TEM analysis (Hitachi electron microscope H-600; Hitachi, Ltd., Tokyo, Japan).

Interfering expression of PLK1 in leukemia cells with RNAi lentivirus. RNAi lentivirus for PLK1 was purchased from Shanghai GeneChem Co, Ltd. RNAi products consist of target-specific lentivirus designed to knock down PLK1 expression sequences are: 5'-CCGAGTTATTCATCGAGAC-3'. The control sequences are: 5'-TTCTCCGAACGTGTCACGT-3'. Lentivirus infection was according to the manufacturer (Shanghai Genechem Co., Ltd.) at a final concentration of 100-200 MOI. PLK1 interference efficiency was measured by western blotting at 3 days after transfection. The rest of the cells were harvested for further analysis.

Cell proliferation analysis. Leukemia cells $\left(2 \times 10^{4}\right)$ were seeded in 96-well plates overnight and incubated with DMSO, or increasing concentrations of RO3280, BI2536 24 to $96 \mathrm{~h}$. Each drug concentration was performed replicated 2 times. Then, $10 \mu$ l Cell Counting Kit-8 (CCK-8; Dojindo Molecular Technologies, Inc., Kumamoto, Japan) solution was added to each well, incubated at $37^{\circ} \mathrm{C}$ for 2 to $4 \mathrm{~h}$ and the optical density (OD) values were measured at $450 \mathrm{~nm}$ using a scanning multi-well spectrophotometer (Bio-Rad Model 550; Bio-Rad Laboratories, Inc., Hercules, CA, USA).

Apoptosis assay. Apoptosis assay was according to the manual operation of BD Annexin V staining kit (cat. no. 556420; BD Biosciences, Franklin Lakes, NJ USA). Briefly, cells were washed 2 times with cold PBS and then were resuspend in 
$1 \mathrm{X}$ binding buffer at a concentration of $\sim 1 \times 10^{6}$ cells $/ \mathrm{ml}$, and $100 \mu \mathrm{l}$ of the solution $\left(\sim 1 \times 10^{5}\right.$ cells $)$ was transfered to a $5-\mathrm{ml}$ culture tube. Annexin V and PI (5 $\mu \mathrm{l} /$ test) was added. Cells were gently mixed and incubated for $15 \mathrm{~min}$ at room temperature in the dark. Four hundred microliters of $1 \mathrm{X}$ binding buffer was added to each tube. Cells were analyzed by flow cytometry within $1 \mathrm{~h} \mathrm{(26).}$

Western blotting. For western blotting, protocol was introduced as described (26). Cellular proteins were blocked and then probed with antibodies against PARP (cat. no. 9542S, 1:1,000), PLK1 (cat.no.4535S, 1:1,000), beclin-1 (cat. no.3495S, 1:1,000), SQSTM1/p62 (cat. no. 8025S, 1:1,000),LC3A/B (cat. no. 4108S, 1:1,000), mTOR (cat. no. 2983S, 1:1,000), phospho-mTOR (Ser2448) (cat. no. 5536S, 1:1,000), adenosine monophosphate-activated protein kinase (AMPK) $\alpha$ (cat. no. 5831S, 1:1,000), phospho-AMPK $\alpha 1$ (Ser485) (cat. no. 2537S, 1:1,000), phospho-AMPK $\alpha 1$ (Thr172) (cat. no. 2537S, 1:1,000), Unc-51-like kinase 1 (ULK1) (cat. no. 6439S, 1:1,000), phospho-ULK1 (Ser317) (cat. no. 12753S, 1:1,000), phospho-ULK1 (Ser757) (cat. no. 14202S, 1:1,000), phospho-ULK1 (Ser555) (cat. no. 5869S, 1:1,000) all these antibodies were purchased from Cell Signaling Technology, Inc. (Danvers, MA, USA) and GAPDH (1:5,000; Sigma-Aldrich). Relative expression of $\mathrm{p}-\mathrm{mTOR} / \mathrm{mTOR}$ was analyzed with Image software according to the user guide.

Patients and samples. Bone marrow specimens were obtained at the time of diagnosis during routine clinical assessment of 140 pediatric patients with AML, who presented at the Department of Hematology and Oncology, Children's Hospital of Soochow University between 2008 and 2013. Ethical approval was provided by the Children's Hospital of Soochow University Ethics Committee (nos. SUEC2008-011 and SUEC2000-021), and informed consent was obtained from the parents or guardians. AML diagnosis was made in accordance with the revised French-American-British (FAB) classification. The main clinical and laboratory features of the patient cohort are summarized in Table I.

Quantitative reverse transcription-polymerase chain reaction (PCR) for PLK1. Quantitative real-time PCR was performed to determine the expression levels of PLK1 genes. Total RNA was reverse transcribed using the Reverse Transcription kit, according to the manufacturer's instructions (Applied Biosystems, Foster City, CA, USA). The real-time PCR primers used to quantify GAPDH expression were forward, 5'-AGAAGGCTGGGGCTCATTTG-3' and reverse, 5'-AGGGGCCATCCACAGTCTTC-3'; and for PLK1 were forward, 5'-CTCAACACGCCTCATCCTC-3' and reverse, 5'-GTGCTCGCTCATGTAATTGC-3'. Expression of PLK1 was normalized to endogenous GAPDH expression.

Statistical analysis. Each experimental condition was performed three times, and these replicates are presented in the results. All values are presented as means \pm SEM. Student's paired t-test was applied to reveal statistical significance. P-values $<0.05$ were considered significant. Statistical analyses were performed using SPSS software for Windows (version 11.5; SPSS, Inc., Chicago, IL, USA).
Table I. Association of PLK1 expression with clinico-pathological characteristics in 140 pediatric AML samples.

\begin{tabular}{|c|c|c|c|c|}
\hline \multirow{2}{*}{$\begin{array}{l}\text { Clinical } \\
\text { variables }\end{array}$} & \multirow{2}{*}{$\begin{array}{c}\text { No. of } \\
\text { patients }\end{array}$} & \multicolumn{2}{|c|}{$\begin{array}{l}\text { PLK1 expression } \\
\text { (n) }\end{array}$} & \multirow[b]{2}{*}{ P-value } \\
\hline & & Low & High & \\
\hline Gender & & & & 0.888 \\
\hline Male & 56 & 34 & 22 & \\
\hline Female & 84 & 50 & 34 & \\
\hline Age (years) & & & & 0.334 \\
\hline$<6$ & 73 & 41 & 32 & \\
\hline$\geq 6$ & 67 & 43 & 24 & \\
\hline Leukocyte $(/ \mu 1)$ & & & & 0.673 \\
\hline$>10,000$ & 83 & 51 & 32 & \\
\hline$\leq 10,000$ & 57 & 33 & 24 & \\
\hline FAB & & & & 0.027 \\
\hline M1-M6 & 123 & 78 & 45 & \\
\hline M7 & 17 & 6 & 11 & \\
\hline Cytogenetics & & & & 0.382 \\
\hline Favorable & 70 & 43 & 27 & \\
\hline Intermediate & 38 & 25 & 13 & \\
\hline Unfavorable & 32 & 16 & 16 & \\
\hline MRD & & & & 0.038 \\
\hline$<0.25 \%$ & 65 & 45 & 20 & \\
\hline$\geq 0.25 \%$ & 75 & 39 & 36 & \\
\hline
\end{tabular}

PLK1, polo-like kinase 1; AML, acute myeloid leukemia; $\mathrm{FAB}$, French-American-British; MRD, minimal residual disease.

\section{Results}

Screening of novel autophagy inducers of AML cells. As autophagy pathway inducers have shown promising effects for treating AML, NB4 AML cells overexpressing LC3-GFP fusion protein were screened with 69 inhibitors $(1 \mu \mathrm{M}$ each; Selleck Chemicals, Houston, TX, USA) for 4 to $12 \mathrm{~h}$ before analysis. LC3 aggregation in the cells was observed during autophagy. LC3-GFP translocation to the autophagic membranes formed bright green fluorescent spots under fluorescence confocal microscopy when the cells underwent autophagy; one spot was equivalent to an autophagosome, which were counted to evaluate the level of autophagy activity (Fig. 1A). Autophagy activity was calculated as autophagy spots per number of cells. Our screening experiment showed that several inhibitors had promising autophagy inducer effects. Autophagy activity $>5$ in the inhibitors BI2536, MLN0905, SK1-I, SBE13 HCL and RO3280 (Fig. 1B-D). Rapamycin was used as the positive control, where its autophagy activity was $\sim 5$. As reported previously, SK1-I is a novel inhibitor of sphingosine kinase 1 (SPHK1) (27), an evolutionarily conserved lipid kinase responsible for converting sphingosine to sphingosine-1-phosphate (S1P). SPHK1 is overexpressed in many cancers and therefore serves as a cancer prognostic biomarker. BI2536, MLN0905, SBE13 HCL and RO3280 
A
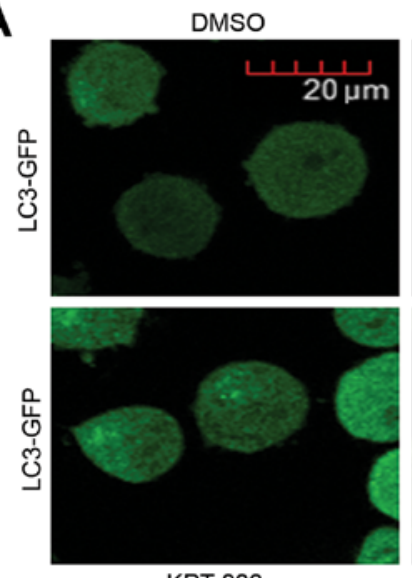

B

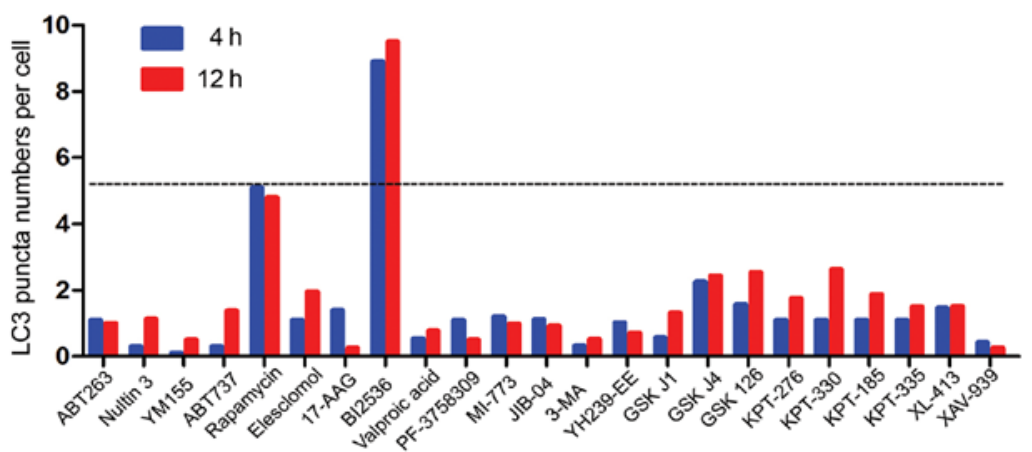

C

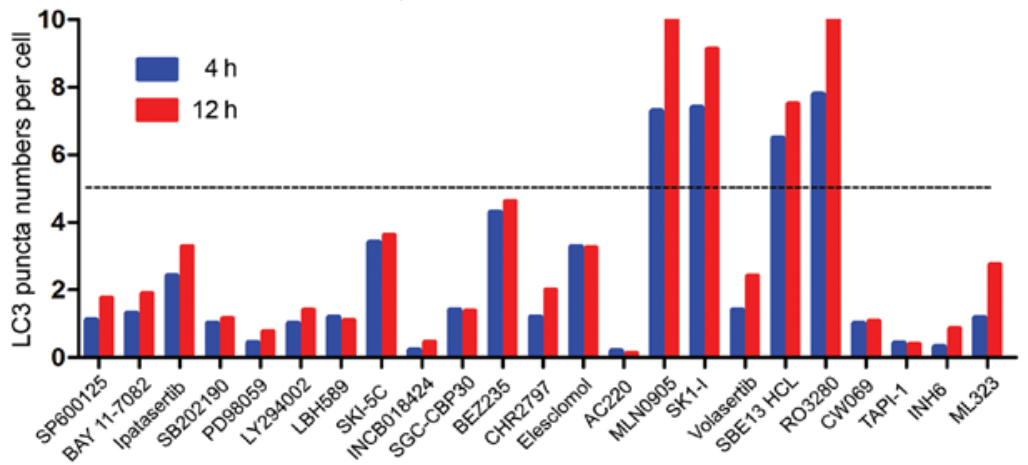

D

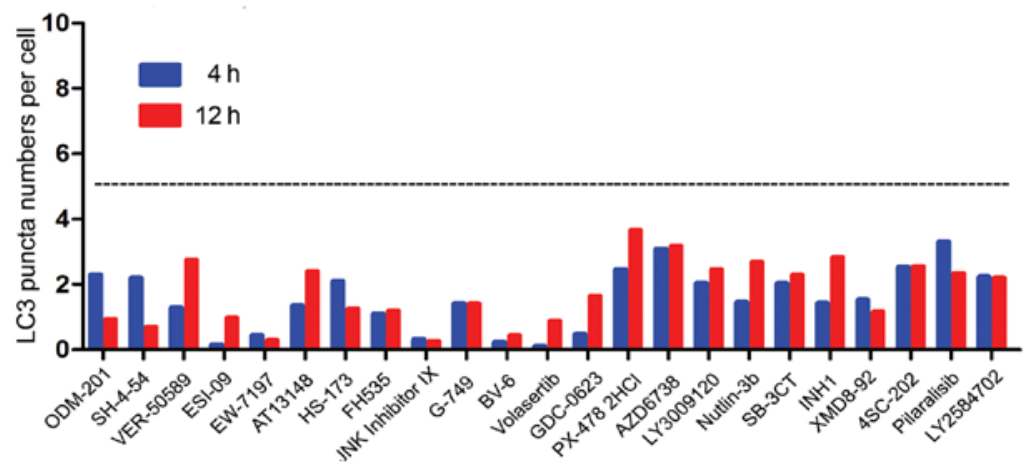

Figure 1. Novel autophagy inducer screen in AML cells. (A) Cells treated with rapamycin (positive control), DMSO (negative control) and 69 inhibitors observed under fluorescence confocal microscopy to analyze autophagy activity. White arrows indicate autophagosomes. (B-D) Autophagy activity was calculated as autophagy spots per number of cells. Our screening experiment showed that several inhibitors had promising autophagy inducer effects. Five-fold change in the figure is marked with dotted line. The autophagy activity of SK1-I, BI2536, MLN0905, SK1-I, SBE13 HCL and RO3280 was >5. AML, acute myeloid leukemia; DMSO, dimethyl sulfoxide.

are all PLK1 inhibitors. PLK1 is always highly expressed in leukemic cell lines and is overexpressed in a majority of samples from patients with AML compared with normal progenitors (14). Yet, its molecular function in the autophagy of AML cells is still unclear.
PLK1 inhibitors induce AML cell autophagy. To confirm the induction of autophagy by the PLK1 inhibitors, we treated NB4 cells with 200 and $500 \mathrm{nM} \mathrm{RO} 3280$ or BI2536 for $4 \mathrm{~h}$ before analysis (Fig. 2A), and found that autophagy activity levels were increased (500 nM BI2536: 9.73 \pm 1.89 vs. DMSO: 0.73 \pm 0.45 , 
A
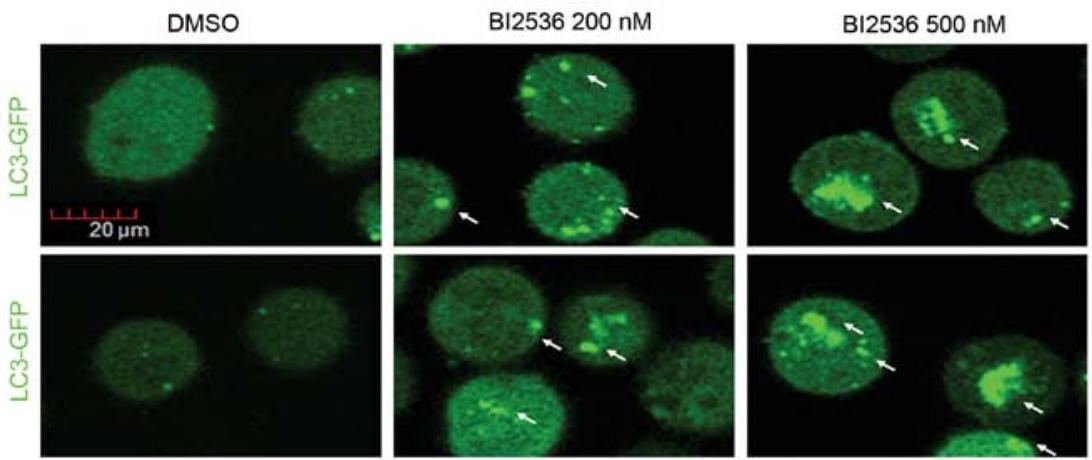

DMSO

$\mathrm{RO} 3280200 \mathrm{nM}$

$\mathrm{RO} 3280500 \mathrm{nM}$
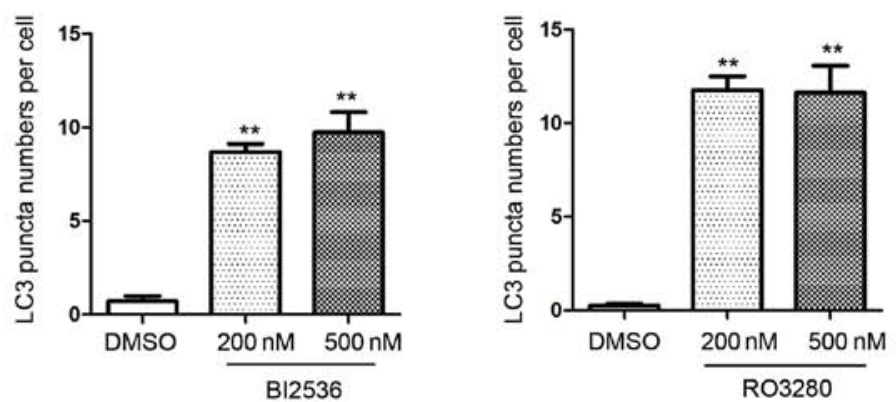

B
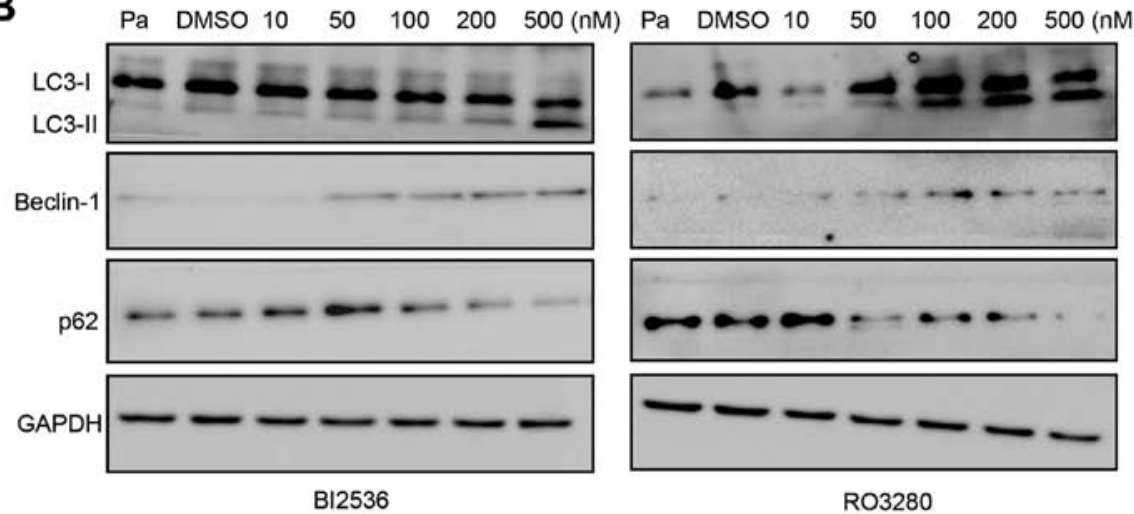

C
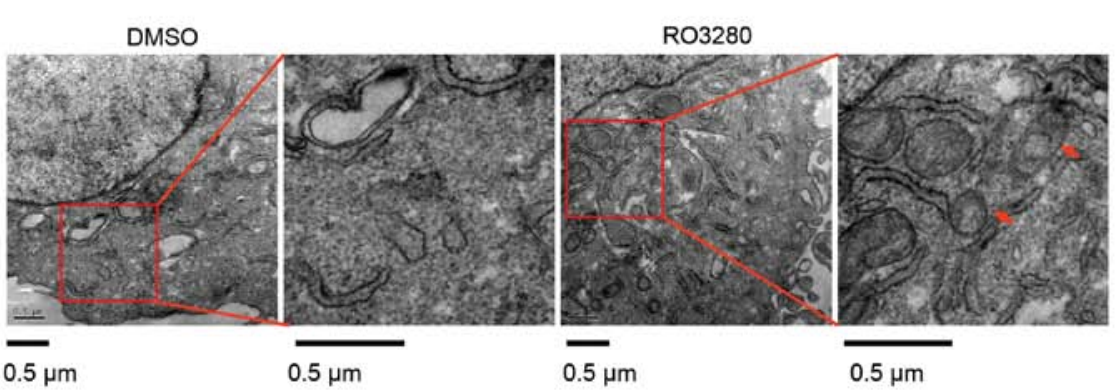

Figure 2. PLK1 inhibitors induce AML cell autophagy. (A) Fluorescence confocal microscopy images of autophagy activity in NB4 cells treated with 200 and $500 \mathrm{nM} \mathrm{RO} 3280$ or BI2536 for $4 \mathrm{~h}$. White arrows indicate autophagosomes. Analysis of autophagy activity in NB4 cells treated with RO3280 or BI2536. ${ }^{* *} \mathrm{p}<0.01$. (B) Western blotting showing more LC3-II in cells treated with RO3280 and BI2536. Beclin-1 was upregulated and SQSTM1/p62 was downregulated in the PLK1 inhibitor group. (C) TEM examination of autophagosomes in cells treated with $500 \mathrm{nM}$ RO3280 for $4 \mathrm{~h}$. Compared with the control cells (left, magnified inset), autophagosomes (red arrows) were rapidly increased in the RO3280-treated NB4 cells (right, magnified inset). PLK1, polo-like kinase 1; AML, acute myeloid leukemia; TEM, transmission electron microscopy.

$\mathrm{p}=0.011 ; 500 \mathrm{nM}$ RO3280: $11.63 \pm 2.48$ vs. DMSO: $0.27 \pm 0.15$, $\mathrm{p}=0.015)$. LC3 is converted from the inactive form (LC3-I) to the cleaved form (LC3-II) during autophagy, rendering LC3 a specific marker for detecting autophagy. Western blotting showed more LC3-II in NB4 cells treated with RO3280 and BI2536. More LC3-II was observed in the 100-500 nM RO3280 group and in the 200-500 nM BI2536 group (Fig. 2B).
Beclin-1 upregulation and SQSTM1/p62 downregulation was also observed in the PLK1 inhibitor treatment groups. TEM was used to examine the autophagosomes in RO3280-treated cells (Fig. 2C). Compared with the DMSO control group, there were more autophagosomes in RO3280-treated NB4 cells. These results are consistent with the autophagy activity analysis involving LC3-GFP. Detecting autophagy is related 
A

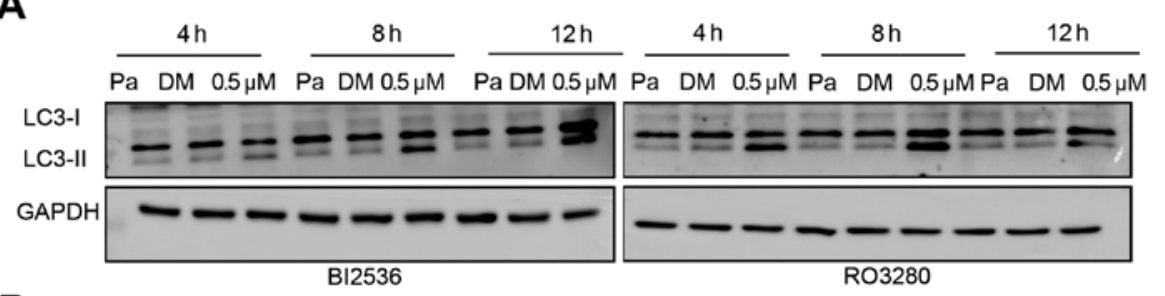

B
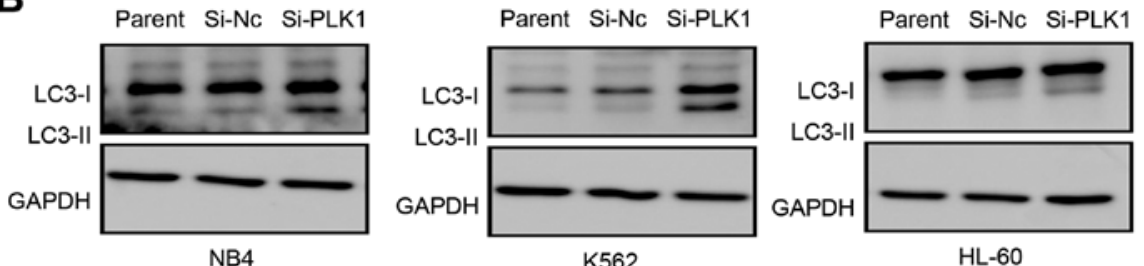

C
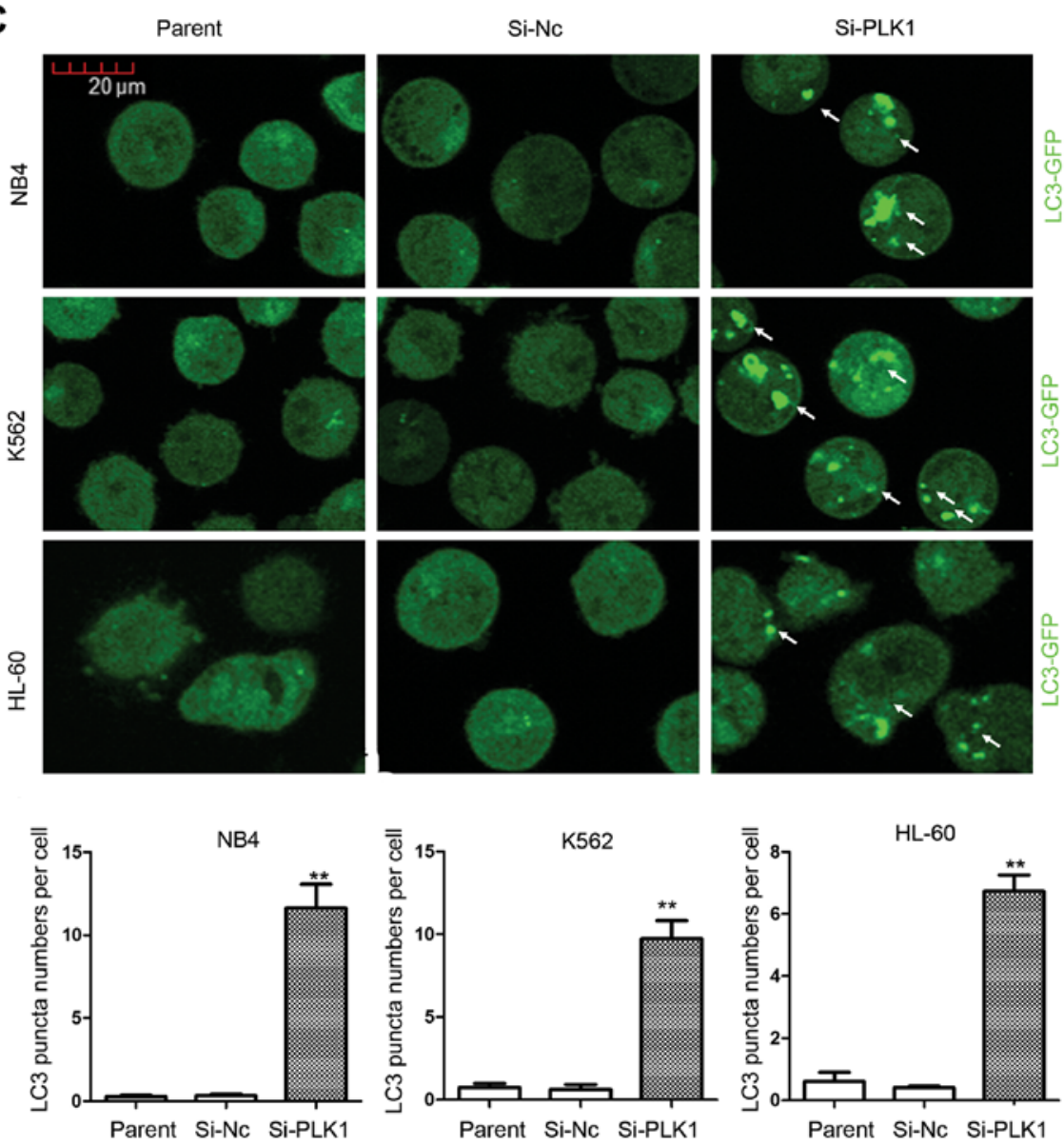

Figure 3. PLK1 inhibition by RNA interference induces more LC3-II in AML cells. (A) NB4 cells were treated with $500 \mathrm{nM}$ RO3280 or BI2536 for 4 to $12 \mathrm{~h}$. There was more LC3-II in NB4 cells treated with $500 \mathrm{nM}$ RO3280 or BI2536. (B) Western blotting of PLK1 and LC3-I/LC3-II in NB4, K562 and HL-60 cells transfected with RNA interference lentivirus. (C) NB4, K562 and HL-60 cells transfected with RNA interference lentivirus observed under fluorescence confocal microscopy for analysis of autophagy activity. White arrows indicate autophagosomes. Autophagy activity in NB4, K562 and HL-60 cells. **p<0.01. PLK1, polo-like kinase 1; AML, acute myeloid leukemia.

to correct timing. We treated NB4 cells with $0.5 \mu \mathrm{M}$ RO3280 or BI2536 continuously 4 to $12 \mathrm{~h}$, and observed their induction of autophagy at different time-points (Fig. 3A). There was more LC3-II in the NB4 cells treated with $0.5 \mu \mathrm{M}$ RO3280 or BI2536. These results suggest that PLK1 inhibitors induce significant AML cell autophagy.

PLK1 inhibition by RNA interference induces AML cell autophagy. Inhibitors tend to have several candidate targets, and their molecular function is influenced by other candidate targets, especially homologous molecules. The molecular function of PLK1 inhibitors is influenced by the candidate targets PLK2, 3 and 4. To confirm the effect of PLK1, we inhibited its expression with RNA interference. When PLK1 expression was downregulated, western blotting detected more LC3-II (Fig. 3B). LC3-GFP analysis showed increased autophagy activity levels when PLK1 expression was significantly downregulated in the three leukemia cell 


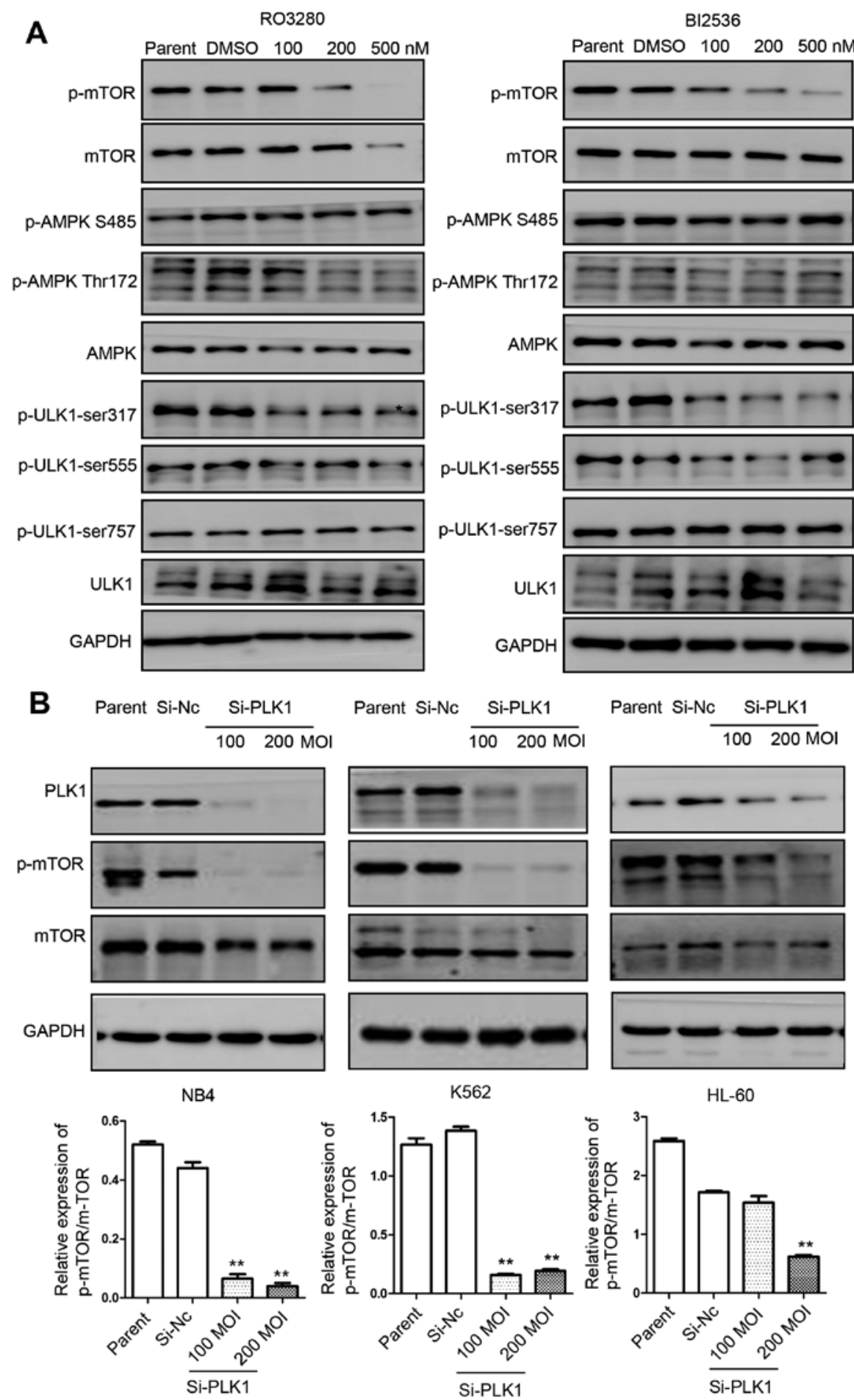

Figure 4. PLK1 inhibition induces AML cell autophagy via mTOR dephosphorylation. (A) Western blotting of mTOR, AMPK and ULK1 expression levels and phosphorylation in NB4 cells treated with RO3280 or BI2536. There was significantly decreased mTOR phosphorylation and ULK1 phosphorylation at Ser317. (B) Western blotting of mTOR expression levels and phosphorylation in NB4, K562 and HL-60 cells transfected with PLK1 RNA interference lentivirus at 100 and 200 MOI. Relative expression of p-mTOR/mTOR was analyzed with ImageJ software according to the user guide. * p $<0.01$. PLK1, polo-like kinase 1; AML, acute myeloid leukemia; mTOR, mammalian target of rapamycin; AMPK, adenosine monophosphate-activated protein kinase; ULK1, Unc-51-like kinase 1.

lines (Fig. 3C). The autophagy activity was calculated and results were as follows: NB4 cells, Si-PLK1: $11.23 \pm 2.43$ vs. Si-Nc: $0.33 \pm 0.15(\mathrm{p}=0.015)$; K562 cells, Si-PLK1: $9.73 \pm 1.89$ vs. Si-Nc: $0.63 \pm 0.49$ ( $\mathrm{p}=0.011)$; HL-60 cells, Si-PLK1: $6.73 \pm 0.90$ vs. Si-Nc: $0.40 \pm 0.10(\mathrm{p}=0.002)$, showing that PLK1 inhibition by RNA interference significantly induces AML cell autophagy (Fig. 3C).
PLK1 inhibition induces AML cell autophagy via mTOR dephosphorylation. We demonstrated that PLK1 inhibition induces significant leukemia cell autophagy. We analyzed the phosphorylation of these three kinases in NB4 cells treated with RO3280 and BI2536, and found that mTOR phosphorylation was reduced significantly in NB4 cells treated with RO3280 and BI2536 (Fig 4A). The dephosphorylation of mTOR is a 
A

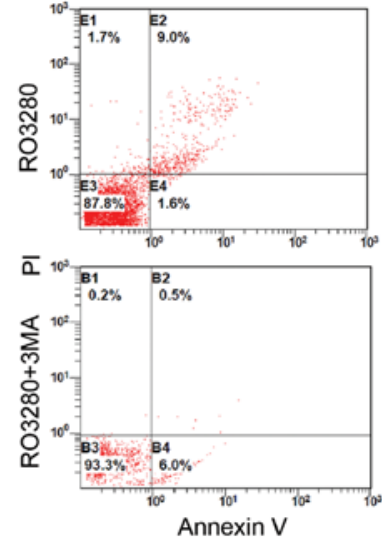

B

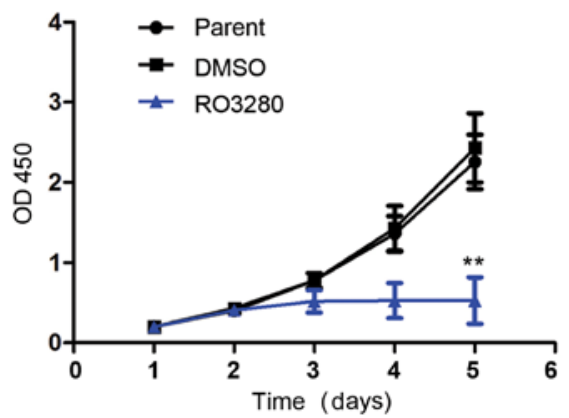

D

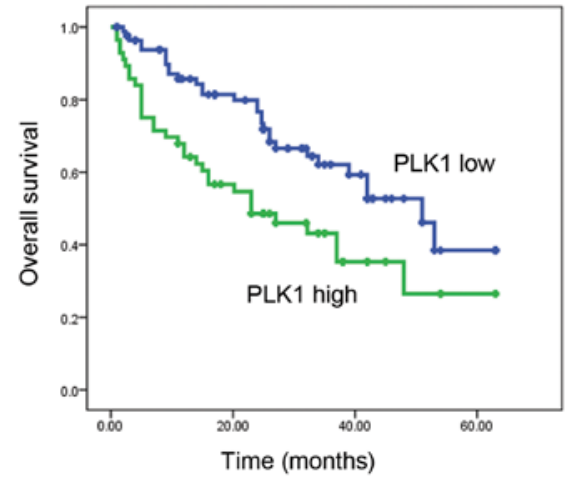

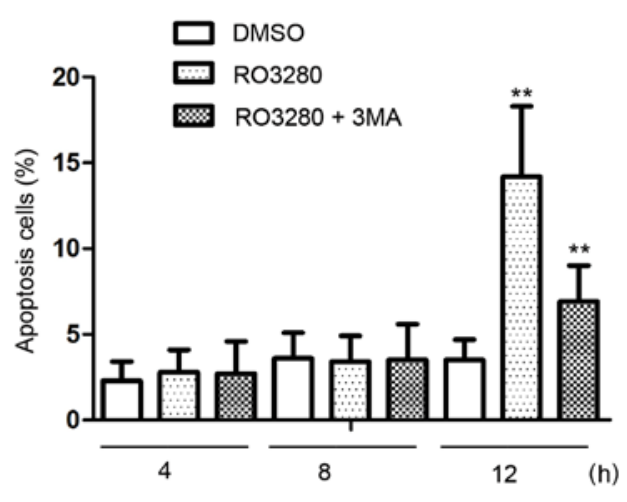

C

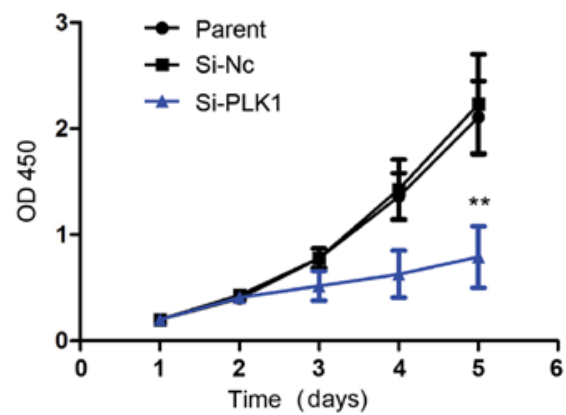

$\mathbf{E}$

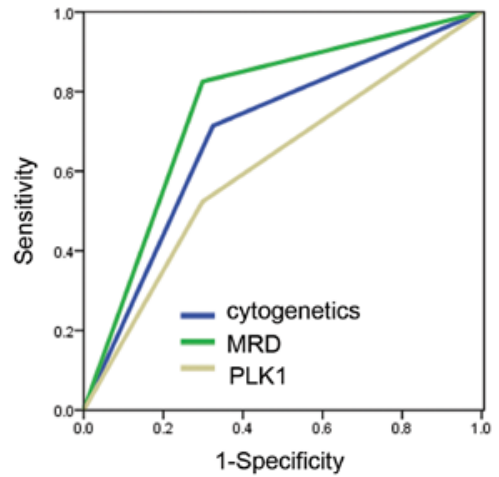

Figure 5. PLK1 is an excellent anticancer target for pediatric AML. (A) Analysis of NB4 cell apoptosis treated with RO3280 or 3-MA. At 4 and 8 h, NB4 cell apoptosis was almost identical between the DMSO and $200 \mathrm{nM} \mathrm{RO} 280$ group. NB4 cell apoptosis increased significantly following 12-h treatment with RO3280, and was inhibited significantly by $5 \mathrm{mM} 3$-MA. ** $<<0.01$. (B) Analysis of NB4 cell proliferation following treatment with $200 \mathrm{nM}$ RO3280. (C) Analysis of NB4 cell proliferation following transfection with PLK1 RNA interference lentivirus at 100 MOI. ${ }^{*}<<0.05$, ${ }^{* *}$ p $<0.01$. (D) The prognostic significance of PLK1 expression was assessed in 140 Chinese patients with pediatric AML with clinical follow-up records. Kaplan-Meier survival analysis revealed shorter survival in the patients with high PLK1 expression in tumors $(\mathrm{p}=0.007)$. (E) ROC curve analysis showing that the area under the curve of cytogenetics, MRD, and PLK1 is 0.695, 0.763 and 0.613, respectively. PLK1, polo-like kinase 1; AML, acute myeloid leukemia; 3-MA, 3-methyladenine; DMSO, dimethyl sulfoxide; MOI, multiplicity of infection; ROC, receiver operating characteristic; MRD, minimal residual disease.

crucial step in the induction of autophagy in eukaryotes. ULK1 phosphorylation at Ser317 was also reduced significantly in NB4 cells treated with RO3280 and BI2536. AMPK levels and AMPK phosphorylation levels did not exhibit significant changes. To confirm the effect of PLK1, RNA interference lentivirus transfection of NB4, K562 and HL-60 cells was used to significantly downregulate PLK1 expression, which was followed by significantly reduced mTOR phosphorylation (Fig. 4B). These results imply that PLK1 inhibition induces AML cell autophagy via mTOR dephosphorylation.

PLK1 is an excellent anticancer target in pediatric AML. PLK1 inhibition induces significant apoptosis in several cancer cell types. The relationship between autophagy and apoptosis following PLK1 inhibition in AML cells remains unclear. Our analysis showed that for 4 and $8 \mathrm{~h}$, NB4 cell apoptosis was almost identical between the DMSO group and $200 \mathrm{nM}$ RO3280 group, suggesting that autophagy occurs earlier than apoptosis when cells are treated with PLK1 inhibitors. Apoptosis increased significantly when the NB4 cells were treated with RO3280 for $12 \mathrm{~h}$, and it was inhibited by $5 \mathrm{mM}$ 3-MA, an autophagy inhibitor (Fig. 5A). Autophagy and apoptosis induced by PLK1 inhibition are closely related, and the specific molecular mechanism warrants further study. Proliferation analysis showed that PLK1 inhibition significantly inhibited NB4 cell proliferation (Fig. 5B and C). 
Table II. Association of PLK1 expression with Kaplan-Meier survival in 140 pediatric AML samples.

\begin{tabular}{lccc}
\hline Variables & $\begin{array}{c}\text { No. of } \\
\text { patients }\end{array}$ & $\begin{array}{c}\text { Over survival } \\
\text { (mean } \pm \text { SE) }\end{array}$ & P-value \\
\hline Cytogenetics & & & $<0.001$ \\
$\quad$ Favorable & 70 & $48.277 \pm 2.857$ & \\
Intermediate & 38 & $34.785 \pm 2.840$ & \\
Unfavorable & 32 & $13.477 \pm 2.245$ & \\
FAB & & & 0.001 \\
M1-M6 & 123 & $40.150 \pm 2.344$ & \\
M7 & 17 & $14.120 \pm 3.343$ & \\
Leukocyte $(\mu 1)$ & & & 0.641 \\
$>10,000$ & 83 & $34.611 \pm 2.472$ & \\
$\leq 10,000$ & 57 & $36.893 \pm 3.431$ & \\
MRD & & & $<0.001$ \\
$<0.25 \%$ & 65 & $53.085 \pm 2.622$ & \\
$\geq 0.25 \%$ & 75 & $25.061 \pm 2.358$ & \\
PLK1 expression & & & 0.007 \\
Low $<10.32 \times 10^{-5}$ & 84 & $42.253 \pm 2.778$ & \\
High $\geq 10.32 \times 10^{-5}$ & 56 & $30.214 \pm 3.521$ & \\
\hline PK1 & & & \\
\hline
\end{tabular}

PLK1, polo-like kinase 1; AML, acute myeloid leukemia; FAB, French-American-British; MRD, minimal residual disease.

Patients were divided into two groups with average value of PLK1 expressions $\left(10.32 \times 10^{-5}\right)$. Results revealed that PLK1 expression was related with FAB subtype and minimal residual disease (MRD; Table I). Kaplan-Meier survival analysis revealed shorter survival in patients with high PLK1 expression in tumors ( $p=0.007$; Table II and Fig. 5D). Multivariate analysis revealed that PLK1 expression is a near independent prognostic factor in pediatric AML patients $(p=0.066$; Table III). Receiver operating characteristic (ROC) curve analysis showed that the area under the curve for cytogenetic, MRD, and PLK1 expression was 0.695, 0.763 and 0.613, respectively (Fig. 5E). In conclusion, our results demonstrate that PLK1 is an excellent anticancer target in pediatric AML.

\section{Discussion}

Autophagy functions as a means of managing cellular stresses and plays a substantial role in tumor cell survival. Decreased autophagic flux results in the development of a myeloproliferative state and AML. In AML, there is copy number loss in the autophagic genes BECN1, ATG7 and ATG5. AML cells often remain sensitive to autophagy-inducing stimuli, leading to the idea that harnessing autophagy can be useful in AML cytotoxic therapy. NB4 AML cells overexpressing LC3-GFP were treated with 69 inhibitors for 4 to $12 \mathrm{~h}$ before analysis, and several had promising autophagy inducer effects, where the autophagy activity of BI2536, MLN0905, SK1-I, SBE13 HCL and RO3280 was $>5$. Very interestingly, these inhibitors all had the same target, PLK1.
Table III. Cox multivariate analysis of PLK1 expression and clinico-pathological features in pediatric AML.

\begin{tabular}{lccc}
\hline Variables & $\begin{array}{c}\text { Odds } \\
\text { ratio }\end{array}$ & $\begin{array}{c}\text { EXP(B) } \\
95 \% \text { CI }\end{array}$ & P-value \\
\hline $\begin{array}{l}\text { Cytogenetics } \\
\text { Favor. vs. intermed. } \\
\text { and unfavorable }\end{array}$ & 7.248 & $2.253(1.247-4.070)$ & 0.007 \\
$\begin{array}{l}\text { MRD } \\
<0.25 \text { vs. } \geq 0.25 \%\end{array}$ & 11.208 & $3.346(1.650-6.786)$ & 0.001 \\
$\begin{array}{l}\text { Leukocyte }(\mu 1) \\
>10,000 \text { vs. } \leq 10,000\end{array}$ & 0.252 & $1.141(0.681-1.912)$ & 0.615 \\
$\begin{array}{l}\text { FAB classification } \\
\text { M7 vs. M1-M6 }\end{array}$ & 6.102 & $2.233(1.180-4.222)$ & 0.014 \\
$\begin{array}{l}\text { PLK1 expression } \\
\text { Low vs. high }\end{array}$ & 3.370 & $1.605(0.968-2.661)$ & 0.066 \\
\hline
\end{tabular}

PLK1, polo-like kinase 1; AML, acute myeloid leukemia; CI, confidence interval; FAB, French-American-British; MRD, minimal residual disease.

PLK1 is expressed during mitosis and is overexpressed in multiple cancers, including acute leukemia (28). PLK1 is also overexpressed in a majority of samples from patients with AML compared with normal progenitors. However, the prognostic value of PLK1 in pediatric AML is still unclear to date. PLK1 supports centrosome functional maturation in late $\mathrm{G}_{2}$ prophase and the establishment of the bipolar spindle. These checkpoints, which occur at $\mathrm{G}_{2} / \mathrm{M}$ transition, are tightly regulated by nuclear serine/threonine kinases, including cyclin-dependent kinases (CDKs), PLKs and Aurora kinases. Evidence suggests that PLK1 can inhibit the transactivation and proapoptotic functions of p53 by physical interaction and phosphorylation (29). Overexpression studies of PLK1 in the NIH3T3 cell line showed that these cells become capable of forming foci and growing in soft agar, and more importantly, can form tumors in nude mice due to PLK1 overexpression (30). In our study, Kaplan-Meier survival analysis revealed shorter survival in patients with high PLK1 expression in tumors. PLK1 inhibition induced significant AML cell apoptosis. These results suggest that PLK1 is an excellent anticancer target in pediatric AML. In this study, we demonstrate that PLK1 inhibition induces AML cell autophagy. Autophagy activity levels were increased when NB4 cells were treated with the PLK1 inhibitors RO3280 and BI2536. Inhibiting PLK1 expression in NB4, K562, and HL-60 cells with RNA interference led to more LC3-II, and autophagy activity was increased when PLK1 expression was significantly downregulated in these cells. Other members of PLK1 family have been reported with a relationship with autophagy. Overexpression of PLK3 mediates the degradation of abnormal prion proteins dependent on chaperone-mediated autophagy (31). PLK2 has a relationship with $\alpha$-synuclein which plays an important role in autophagy (32). These results indicate that PLK1 family members may all have an important role in autophay. Also, this is the first study of autophagy induction in AML cells following PLK1 inhibition. 
To date, the molecular mechanism of PLK1 inhibition in the induction of AML cell autophagy remains unknown. mTOR, or FK506-binding protein 12 (FKBP12)-rapamycinassociated protein 1 , is a serine/threonine protein kinase from the family of PI3K-related kinases. In mammals, amino acid sensing and other signals such as reactive oxygen species (ROS) and growth factors regulate the activity of the protein kinases mTOR and AMPK, which regulate autophagy through inhibitory phosphorylation of ULK1 and 2. Induction of autophagy results in ULK kinase dephosphorylation and activation. The active ULK relocalizes to the site of autophagosome initiation, contributing to activation of the downstream autophagy components. Accordingly, mTOR, AMPK and ULK1 are important regulators of autophagy initiation. As reported previously, under nutrient sufficiency, high mTOR activity prevents ULK1 activation by phosphorylating and disrupting the interaction between ULK1 and AMPK (33-35). This coordinated phosphorylation is important for ULK1 in autophagy induction. We analyzed the protein phosphorylation of mTOR, AMPK and ULK1, three important regulators of autophagy, and found that mTOR phosphorylation was reduced significantly in NB4 cells treated with RO3280 and BI2536. To confirm the effect of PLK1 in NB4, K562 and HL-60 cells, mTOR phosphorylation was also reduced significantly when PLK1 expression was downregulated using RNA interference. mTOR, a serine/threonine protein kinase with a molecular size of $\sim 300 \mathrm{kDa}$, belongs to the phosphatidylinositol 3-kinase-related kinase (PIKK) family. Its activity is inhibited under nutrient starvation and other stimuli of autophagy induction, and its dephosphorylation is a crucial step in the induction of autophagy in eukaryotes. The PI3K/AKT/mTOR pathway is very important in cell growth, survival, apoptosis, angiogenesis and phagocytosis. Disorder of this pathway results in disease, including cancer, neurological disease and autoimmune disease. The $\mathrm{PI} 3 \mathrm{~K} / \mathrm{AKT} / \mathrm{mTOR}$ pathway is constitutively activated in $\sim 50-80 \%$ of AML cases (36), and its activation is associated with poor prognosis of AML. PI3K/AKT/mTOR may be an important therapeutic target in AML. Unfortunately, targeting the mTOR pathway with single-agent rapamycin in clinical trials was unsuccessful (37). As an alternative to adding chemotherapy, it is useful to target both the mTOR pathway and other cancer targets to generate more effective responses $(38,39)$. As PLK1 inhibitors inhibit the activity of PLK1 and mTOR, two important pathways in AML, the present findings imply that PLK1 inhibitors may have good clinical application in AML. The details of PLK1 regulation of mTOR phosphorylation are still unknown and warrant further studies.

In conclusion, we demonstrate that PLK1 is an excellent anticancer target in pediatric AML; its inhibition induces autophagy of AML cells and results in mTOR dephosphorylation in AML cells. These results may provide new insights into the molecular mechanism of PLK1 in autophagy regulation.

\section{Acknowledgements}

This study was supported by grants from National Natural Science Foundation (81570125, 81370627, 81300423, $81502500,81501703,81501840,81502157,81501700$ and
31500718), Natural Science Foundation of Jiangsu Province (BK20151207, H201420), Key Medical Subjects of Jiangsu Province (XK201120), Innovative Team of Jiangsu Province (LJ201114, LJ201126), Special Clinical Medical Science and Technology of Jiangsu Province (BL2012050, BL2013014, BL2012051), Major Scientific and Technological Special Project for 'Significant New Drugs Creation' (2012ZX09103301-040). This manuscript was copy-edited by an English speaking professional with science background at Elixigen Corporation (Huntington Beach, CA, USA).

\section{References}

1. Watson AS, Riffelmacher T, Stranks A, Williams O, De Boer J, Cain K, MacFarlane M, McGouran J, Kessler B, Khandwala S, et al: Autophagy limits proliferation and glycolytic metabolism in acute myeloid leukemia. Cell Death Discov 1: 15008,2015

2. Radwan SM, Hamdy NM, Hegab HM and El-Mesallamy HO: Beclin-1 and hypoxia-inducible factor- $1 \alpha$ genes expression: potential biomarkers in acute leukemia patients. Cancer Biomark 16: 619-626, 2016

3. Hu B, Yue QF, Chen Y, Bu FD, Sun CY and Liu XY: Expression of autophagy related gene BECLIN-1 and number of autophagic vacuoles in bone marrow mononuclear cells from 40 myelodysplastic syndromes patients and their significance. Zhongguo Shi Yan Xue Ye Xue Za Zhi 23: 146-149, 2015 (In Chinese).

4. Piya S, Kornblau SM, Ruvolo VR, Mu H, Ruvolo PP, McQueen T, Davis RE, Hail N Jr, Kantarjian H, Andreeff M, et al: Atg7 suppression enhances chemotherapeutic agent sensitivity and overcomes stroma-mediated chemoresistance in acute myeloid leukemia. Blood 128: 1260-1269, 2016.

5. Kim Y, Eom JI, Jeung HK, Jang JE, Kim JS, Cheong JW, Kim YS and Min YH: Induction of cytosine arabinoside-resistant human myeloid leukemia cell death through autophagy regulation by hydroxychloroquine. Biomed Pharmacother 73: 87-96, 2015.

6. Zare-Abdollahi D, Safari S, Movafagh A, Ghadiani M, Tabarraee M, Riazi-Isfahani S, Gorji S, Keyvan L and Gachkar L: Expression analysis of BECN1 in acute myeloid leukemia: association with distinct cytogenetic and molecular abnormalities. Int J Lab Hematol 38: 125-132, 2016.

7. Bertacchini J, Heidari N, Mediani L, Capitani S, Shahjahani M, Ahmadzadeh A and Saki N: Targeting PI3K/AKT/mTOR network for treatment of leukemia. Cell Mol Life Sci 72: 2337-2347, 2015.

8. Zeng Z, Wang RY, Qiu YH, Mak DH, Coombes K, Yoo SY, Zhang Q, Jessen K, Liu Y, Rommel C, et al: MLN0128, a novel mTOR kinase inhibitor, disrupts survival signaling and triggers apoptosis in AML and AML stem/progenitor cells. Oncotarget 7: 55083-55097, 2016.

9. Lindblad O, Cordero E, Puissant A, Macaulay L, Ramos A, Kabir NN, Sun J, Vallon-Christersson J, Haraldsson K, Hemann MT, et al: Aberrant activation of the PI3K/mTOR pathway promotes resistance to sorafenib in AML. Oncogene 35: 5119-5131, 2016.

10. Maire V, Némati F, Richardson M, Vincent-Salomon A, Tesson B, Rigaill G, Gravier E, Marty-Prouvost B, De Koning L, Lang G, et al: Polo-like kinase 1: a potential therapeutic option in combination with conventional chemotherapy for the management of patients with triple-negative breast cancer. Cancer Res 73: 813-823, 2013.

11. Deeraksa A, Pan J, Sha Y, Liu XD, Eissa NT, Lin SH and Yu-Lee LY: Plk1 is upregulated in androgen-insensitive prostate cancer cells and its inhibition leads to necroptosis. Oncogene 32: 2973-2983, 2013

12. Zhang G, Zhang Z and Liu Z: Polo-like kinase 1 is overexpressed in renal cancer and participates in the proliferation and invasion of renal cancer cells. Tumour Biol 34: 1887-1894, 2013.

13. Ackermann S, Goeser F, Schulte JH, Schramm A, Ehemann V, Hero B, Eggert A, Berthold F and Fischer M: Polo-like kinase 1 is a therapeutic target in high-risk neuroblastoma. Clin Cancer Res 17: 731-741, 2011.

14. Renner AG, Dos Santos C, Recher C, Bailly C, Créancier L, Kruczynski A, Payrastre B and Manenti S: Polo-like kinase 1 is overexpressed in acute myeloid leukemia and its inhibition preferentially targets the proliferation of leukemic cells. Blood 114: 659-662, 2009 
15. Zhang Y, Du XL, Wang CJ, Lin DC, Ruan X, Feng YB, Huo YQ, Peng H, Cui JL, Zhang TT, et al: Reciprocal activation between PLK1 and Stat3 contributes to survival and proliferation of esophageal cancer cells. Gastroenterology 142: 521-530, 2012.

16. Behren A, Mühlen S, Acuna Sanhueza GA, Schwager C, Plinkert PK, Huber PE, Abdollahi A and Simon C: Phenotype-assisted transcriptome analysis identifies FOXM1 downstream from Ras-MKK3-p38 to regulate in vitro cellular invasion. Oncogene 29: 1519-1530, 2010.

17. Valsasina B, Beria I, Alli C, Alzani R, Avanzi N, Ballinari D, Cappella P, Caruso M, Casolaro A, Ciavolella A, et al: NMS-P937, an orally available, specific small-molecule polo-like kinase 1 inhibitor with antitumor activity in solid and hematologic malignancies. Mol Cancer Ther 11: 1006-1016, 2012

18. Hikichi Y, Honda K, Hikami K, Miyashita H, Kaieda I, Murai S, Uchiyama N, Hasegawa M, Kawamoto T, Sato T, et al: TAK-960, a novel, orally available, selective inhibitor of polo-like kinase 1 , shows broad-spectrum preclinical antitumor activity in multiple dosing regimens. Mol Cancer Ther 11: 700-709, 2012.

19. Chen S, Bartkovitz D, Cai J, Chen Y, Chen Z, Chu XJ, Le K, Le NT, Luk KC, Mischke S, et al: Identification of novel, potent and selective inhibitors of polo-like kinase 1. Bioorg Med Chem Lett 22: 1247-1250, 2012

20. Gumireddy K, Reddy MV, Cosenza SC, Boominathan R, Baker SJ, Papathi N, Jiang J, Holland J and Reddy EP: ON01910, a non-ATP-competitive small molecule inhibitor of Plk1, is a potent anticancer agent. Cancer Cell 7: 275-286, 2005.

21. Talati C, Griffiths EA, Wetzler M and Wang ES: Polo-like kinase inhibitors in hematologic malignancies. Crit Rev Oncol Hematol 98: 200-210, 2016.

22. Hao Z and Kota V: Volasertib for AML: clinical use and patient consideration. Onco Targets Ther 8: 1761-1771, 2015.

23. Gutteridge RE, Ndiaye MA, Liu X and Ahmad N: Plk1 inhibitors in cancer therapy: from laboratory to clinics. Mol Cancer Ther 15: 1427-1435, 2016

24. Feng L, Ma Y, Sun J, Shen Q, Liu L, Lu H, Wang F, Yue Y, Li J, Zhang S, et al: YY1-MIR372-SQSTM1 regulatory axis in autophagy. Autophagy 10: 1442-1453, 2014.

25. Wang KF, Yang H, Jiang WQ, Li S and Cai YC: Puquitinib mesylate (XC-302) induces autophagy via inhibiting the $\mathrm{PI} 3 \mathrm{~K} / \mathrm{AKT} / \mathrm{mTOR}$ signaling pathway in nasopharyngeal cancer cells. Int J Mol Med 36: 1556-1562, 2015.

26. Wang NN, Li ZH, Zhao H, Tao YF, Xu LX, Lu J, Cao L, Du XJ, Sun LC, Zhao WL, et al: Molecular targeting of the oncoprotein PLK1 in pediatric acute myeloid leukemia: RO3280, a novel PLK1 inhibitor, induces apoptosis in leukemia cells. Int J Mol Sci 16: 1266-1292, 2015.

27. Price MM, Oskeritzian CA, Falanga YT, Harikumar KB, Allegood JC, Alvarez SE, Conrad D, Ryan JJ, Milstien S and Spiegel S: A specific sphingosine kinase 1 inhibitor attenuates airway hyperresponsiveness and inflammation in a mast cell-dependent murine model of allergic asthma. J Allergy Clin Immunol 131: 501-511, 2013.
28. Gjertsen BT and Schöffski P: Discovery and development of the polo-like kinase inhibitor volasertib in cancer therapy. Leukemia 29: 11-19, 2014

29. Liu X and Erikson RL: Polo-like kinase (Plk)1 depletion induces apoptosis in cancer cells. Proc Natl Acad Sci USA 100: 5789-5794, 2003

30. Malumbres M and Barbacid M: Cell cycle kinases in cancer. Curr Opin Genet Dev 17: 60-65, 2007.

31. Wang H, Tian C, Sun J, Chen LN, Lv Y, Yang XD, Xiao K, Wang J, Chen C, Shi Q, et al: Overexpression of PLK3 mediates the degradation of abnormal prion proteins dependent on chaperone-mediated autophagy. Mol Neurobiol: Jun 25, 2016 (Epub ahead of print)

32. Kim T, Mehta SL, Kaimal B, Lyons K, Dempsey RJ and Vemuganti R: Poststroke Induction of $\alpha$-synuclein mediates ischemic brain damage. J Neurosci 36: 7055-7065, 2016.

33. Petherick KJ, Conway OJ, Mpamhanga C, Osborne SA, Kamal A, Saxty B and Ganley IG: Pharmacological inhibition of ULK1 kinase blocks mammalian target of rapamycin (mTOR)-dependent autophagy. J Biol Chem 290: 28726, 2015.

34. Fan XY, Tian C, Wang H, Xu Y, Ren K, Zhang BY, Gao C, Shi Q, Meng G, Zhang LB, et al: Activation of the AMPK-ULK1 pathway plays an important role in autophagy during prion infection. Sci Rep 5: 14728, 2015.

35. Basu S, Rajakaruna S, Reyes B, Van Bockstaele E and Menko AS: Suppression of MAPK/JNK-MTORC1 signaling leads to premature loss of organelles and nuclei by autophagy during terminal differentiation of lens fiber cells. Autophagy 10: 1193-1211, 2014

36. Evangelisti C, Evangelisti C, Bressanin D, Buontempo F Chiarini F, Lonetti A, Soncin M, Spartà A, McCubrey JA and Martelli AM: Targeting phosphatidylinositol 3-kinase signaling in acute myelogenous leukemia. Expert Opin Ther Targets 17: 921-936, 2013

37. Callera F, Lopes CO, Rosa ES and Mulin CC: Lack of antileukemic activity of rapamycin in elderly patients with acute myeloid leukemia evolving from a myelodysplastic syndrome. Leuk Res 32: 1633-1634, 2008.

38. Zou H, Li L, Garcia Carcedo I, Xu ZP, Monteiro M and $\mathrm{Gu}$ W: Synergistic inhibition of colon cancer cell growth with nanoemulsion-loaded paclitaxel and PI3K/mTOR dual inhibitor BEZ235 through apoptosis. Int J Nanomed 11: 1947-1958, 2016.

39. Park HS, Hong SK, Oh MM, Yoon CY, Jeong SJ, Byun SS, Cheon J, Lee SE and Moon G: Synergistic antitumor effect of NVP-BEZ235 and sunitinib on docetaxel-resistant human castration-resistant prostate cancer cells. Anticancer Res 34: 3457-3468, 2014. 